\title{
Parametric Channel Estimation for Pseudo-Random User-Allocation in Uplink OFDMA
}

\author{
Lior Eldar* \\ M. R. Raghavendra \\ S. Bhashyam \\ Ron Bercovich* \\ K. Giridhar \\ Telecommunications and Computer Networks (TeNeT) Group, \\ Department of Electrical Engineering, Indian Institute of Technology Madras 600036, India. \\ [raghumr, srikrishna, giri]@tenet.res.in \\ *Wireless Infrastructure Department, \\ Freescale Semiconductor Israel Ltd., Hertzliya 46725, Israel. \\ rm96491@freescale.com
}

\begin{abstract}
In this work, we present an algorithm for parametric estimation of a wireless channel for OFDMA transmission, tailored to the pseudo-random "tile" allocation pattern prevalent in multi-user allocation schemes ${ }^{1}$. Such tile allocations are usually common in the uplink where a tile is usually a small number of adjacent data subcarriers with a few pilot subcarriers. The algorithm estimates the delay subspace of the parametric channel description, and shows robustness for high RMS delay-spread channels. The estimator error convergence performance improves with increase in the channel Doppler frequency. Although the proposed algorithm requires more intensive computation than straight-forward intra-tile linear interpolation, it offers a greatly enhanced Bit-Error-Rate (BER) performance. In mobile channel environments, the need for numerous re-transmissions is therefore decreased, making this algorithm suitable for low BER applications such as video and data.
\end{abstract}

\section{INTRODUCTION}

Multi-user allocation schemes in OFDM transmission are becoming increasingly complex, with the need for greater flexibility in the allocation of transmission resources. The increased flexibility is necessary for simultaneous satisfaction of competing transmission needs of various applications requiring different qualities of service. The complexity of such schemes is further increased with the employment of pseudo-random resource allocation techniques introduced to recent wireless standards (such as IEEE 802.16d/e) in order to provide protection against selective fading that harms certain users more than their share.

The downside of such complex allocation schemes is the increased difficulty in estimating the channel response: the irregular distribution of channel information (implicitly held by pilots) over the available bandwidth hinders the estimation of statistical properties of the channel, especially the frequency auto-correlation matrix. Hence, the standards have resorted to supplying local channel information by embedding pilots in each chunk of allocated bandwidth. However, the local channel information, in the absence of more global channel information (such as correlation) is limited in its performance. In fact, in highly selective multi-path fading channels found in

\footnotetext{
${ }^{1}$ The method described in the article is protected by patent no. PCT/IB2005/052954 - filed at WIPO
}

hilly terrain [11] scenarios for example, non-statistical linear interpolation of the channel response at data locations may prove futile.

Previous research [6], [7] has shown that parametric channel estimation can greatly reduce the channel estimation error in sparse wireless channels. The work in [6] has relied on the assumption of evenly spaced pilot locations in frequency domain to enable ESPRIT (Estimation of Signal Parameters via Rotational Invariance Technique)-based parametric estimation. In this paper, we relax the restriction of equal spacing between pilots to support the usage of ESPRIT-based [10] parametric estimation in environments of irregular pilot distribution. By this method, we are able to estimate the location of the channel taps within a marginal error, and as a result, decrease the estimation error to more than $8 \mathrm{~dB}$ below the signal to noise ratio (SNR) level.

\section{A. Basic Notation}

In this paper, bold face letters denote vectors or matrices; $(.)^{T},(.)^{*}$ and $(.)^{H}$ denote transpose, complex conjugate, Hermitian respectively; $\mathbf{0}$ denotes the zero column vector unless its size mentioned; $\mathbf{e}_{p}$ denotes the column vector whose $p^{\text {th }}$ entry is 1 and other entries are 0 's; $\mathcal{S}\{\mathbf{X}\}$ denotes the span of the columns of matrix $\mathbf{X} ;\lceil x\rfloor$ denotes the integer nearest to $x ; \bigcup_{i=1}^{k} \mathbf{x}_{i}$ denotes the union of $\mathbf{x}_{1}, \mathbf{x}_{2}, \ldots, \mathbf{x}_{k}$.

\section{OFDMA SYSTEM MODEL}

\section{A. Channel model}

Consider an OFDMA system with $M$ active users sharing a bandwidth of $B=\frac{1}{T} \mathrm{~Hz}$ ( $T$ is the sampling period) as shown in Fig. 1. The system consists of $K$ subcarriers of which $K_{u}$ are useful subcarriers (excluding guard bands and DC subcarrier). The users are allocated non-overlapping subcarriers in the spectrum depending on their needs.

The discrete time baseband channel consists of $L$ multipath components and has the form

$$
h(l)=\sum_{m=0}^{L-1} h_{m} \delta\left(l-l_{m}\right)
$$




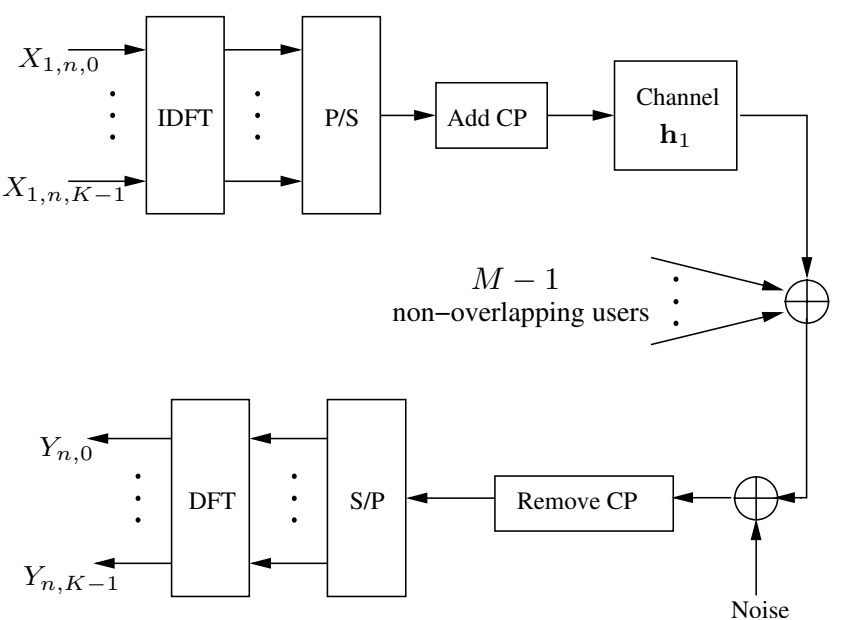

Fig. 1. Discrete time baseband equivalent of an OFDMA system with $M$ users

where $h_{m}$ is a zero-mean complex Gaussian random variable with $E\left[h_{i} h_{j}^{*}\right]=0$ for $i \neq j$. In frequency domain

$$
\mathbf{H}=\mathbf{F h}
$$

where $\mathbf{H}=\left[H_{0}, H_{1}, \ldots, H_{K-1}\right]^{T}, \mathbf{h}=\left[h_{0}, \ldots, h_{L-1}, 0, \ldots, 0\right]^{T}$ and $\mathbf{F}$ is $K$ point DFT matrix. The impulse response length $l_{L-1}$ is the upper bounded by length of cyclic prefix $\left(L_{c p}\right)$.

The received signal in frequency domain is written as,

$$
\mathbf{Y}_{n}=\sum_{i=1}^{M} \mathbf{X}_{i, n} \mathbf{H}_{i, n}+\mathbf{V}_{n}
$$

where $\mathbf{X}_{i, n}=\operatorname{diag}\left(X_{i, n, 0}, \ldots, X_{i, n, K-1}\right)$ is $K \times K$ diagonal data matrix and $\mathbf{H}_{i, n}$ is the $K \times 1$ channel vector (2) corresponding to the $i^{t h}$ user in $n^{\text {th }}$ symbol. The noise vector $\mathbf{V}_{n}$ is distributed as $\mathbf{V}_{n} \sim \mathcal{C N}\left(0, \sigma^{2} \mathbf{I}_{K}\right)$. In further discussions, we assume the first user to be the desired user and drop the user specific subscripts.

In this paper, we have considered an OFDMA system operating in partially used sub-channeling (PUSC) mode akin to the IEEE 802.16d/e WMAN project [1], [2] with $K=2048$ and $K_{u}=1680$. The frame structure is briefly described here. Three consecutive OFDM symbols in time forms a "slot". We reference the three OFDM symbols in the $q^{\text {th }}$ slot as $q_{1}, q_{2}$, and $q_{3}$ respectively. The useful subcarriers of a slot are divided into the "tiles". A tile is defined as a band of 4 frequency subcarriers by 3 time symbols, containing 4 pilots at its corners. The uplink PUSC tile structure is as shown in the Fig. 2.

The allocation of tiles to the users is as follows: In each time slot ( 3 OFDM symbols in time) there are $\frac{3 K_{u}}{12}=420$ tiles. From each group of 70 contiguous tiles ( 6 groups altogether) a tile is selected at random to form a "subchannel". That is, 6 randomly selected tiles, one from each group, make up a single subchannel. The number of subchannels allocated to a single user can be any integer in $(1,2, \ldots, 70)$.

Let $N_{s}$ be the number of subchannels allocated for the desired user in a slot. We assume that the subchannel allo-

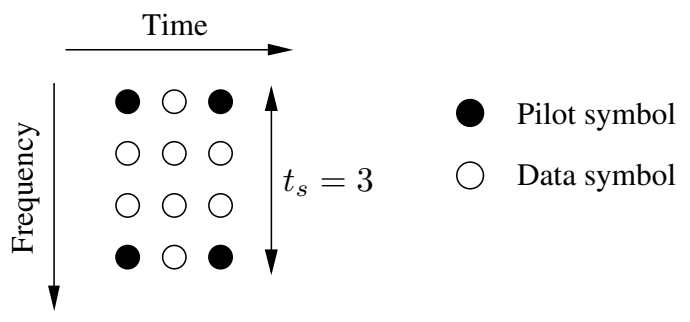

Fig. 2. IEEE $802.16 \mathrm{~d} / \mathrm{e}$ uplink tile structure

cation does not change over the duration of multipath delay estimation. Let $\mathcal{I}$ represent the set of all subcarrier indices of the desired user. The number of pilot subcarriers allocated for the desired user in an OFDM symbol $\left(1^{\text {st }}\right.$ or $3^{\text {rd }}$ symbol in a slot) is $K_{p}=N_{s} \times 6 \times 2$ (this follows from tile structure given in Fig. 2). Note that $K_{p}$ is always an even number. Let $\mathcal{I}_{p}$ represent the set of all pilot subcarrier indices of the desired user in the $1^{\text {st }}$ (or $3^{\text {rd }}$ ) OFDM symbol.

We address channel estimation applied to the uplink transmission scenario, in which the channel estimates are derived from pilots embedded in the transmission sequence. The tile structure shown in Fig. 2 will serve as the basis for this discussion. While intra-tile linear interpolation is a natural choice in this case, as the pilots "surround" the data carriers in both frequency and time dimensions, it suffers from an inherent interpolation error floor, which becomes acute in high RMS-delay spread channels (SUI models 5,6 for instance). In order to break the error floor, inter-tile processing is necessary.

The proposed channel estimator exploits the structure of the tile as a sensor "doublet" (taken from the terminology of [10]) to estimate the tap locations. After estimating the tap locations on the timescale within a certain error range, the algorithm attempts to localize the tap locations to a single sample, and finally compute the amplitude at these tap locations.

\section{Channel Estimation}

The received signal in frequency domain on pilot subcarriers of the desired user is written as (3)

$$
\mathbf{Y}_{n}^{\prime}=\mathbf{X}_{n}^{\prime} \mathbf{H}_{n}^{\prime}+\mathbf{V}_{n}^{\prime}
$$

With the linear, Gaussian measurement model in (4) all linear unbiased channel estimators lead to least squares (LS) estimator [3], [5] and is given by,

$$
\hat{\mathbf{H}}_{l s, n}^{\prime}=\mathbf{X}_{n}^{\prime-1} \mathbf{Y}_{n}^{\prime}=\mathbf{F}^{\prime} \mathbf{h}_{n}+\underbrace{\mathbf{X}_{n}^{\prime-1} \mathbf{V}_{n}^{\prime}}_{\mathbf{W}_{n}^{\prime}}
$$

where $\mathbf{F}^{\prime}$ is the modified matrix obtained from the $K$-point DFT matrix by selecting the rows corresponding to the pilot subcarrier indices $\mathcal{I}_{p}$ and the noise vector is distributed as $\mathbf{W}_{n}^{\prime} \sim \mathcal{C N}\left(0, \sigma^{2} \mathbf{I}_{K_{p}}\right)$ (The pilot symbols are assumed to be BPSK modulated with $\left|X_{n}^{\prime}\right|^{2}=1$ ).

The proposed parametric channel estimation method exploits the shift invariance structure of the signal space spanned by the channel estimates corresponding to the even and odd 
pilot locations of $\hat{\mathbf{H}}_{l s, n}^{\prime}$ and hence enabling the use of ESPRITbased multipath delay estimator.

The elements of the vector $\hat{\mathbf{H}}_{l s, n}^{\prime}$ are rearranged to give the augmented $\hat{\mathbf{H}}_{a u g, n}^{\prime}$ as follows:

$$
\hat{\mathbf{H}}_{a u g, n}^{\prime}=\left[\begin{array}{c}
\hat{\mathbf{H}}_{o, n}^{\prime} \\
\hat{\mathbf{H}}_{e, n}^{\prime}
\end{array}\right]=\mathbf{H}_{a u g, n}^{\prime}+\mathbf{W}_{a u g, n}^{\prime}
$$

where $\hat{\mathbf{H}}_{o, n}^{\prime}, \hat{\mathbf{H}}_{e, n}^{\prime}$ represent the odd and even elements of the vector $\hat{\mathbf{H}}_{l s, n}^{\prime}$ respectively, and $\mathbf{W}_{a u g, n}^{\prime}$ represent the corresponding rearrangement in the noise vector $\mathbf{W}_{n}^{\prime}$. The vectors $\hat{\mathbf{H}}_{o, n}^{\prime}, \hat{\mathbf{H}}_{e, n}^{\prime}$ are derived as

$$
\hat{\mathbf{H}}_{o, n}^{\prime}=\mathbf{J}_{1} \hat{\mathbf{H}}_{l s, n}^{\prime} \quad \text { and } \quad \hat{\mathbf{H}}_{e, n}^{\prime}=\mathbf{J}_{2} \hat{\mathbf{H}}_{l s, n}^{\prime}
$$

where $\mathbf{J}_{1}$ and $\mathbf{J}_{2}$ are $\frac{K_{p}}{2} \times K_{p}$ selection matrices defined as

$$
\begin{aligned}
& \mathbf{J}_{1}=\left[\begin{array}{lllllll}
\mathbf{e}_{1} & \mathbf{0} & \mathbf{e}_{2} & \mathbf{0} & \mathbf{e}_{3} & \ldots & \mathbf{0}
\end{array}\right] \\
& \mathbf{J}_{2}=\left[\begin{array}{lllllll}
\mathbf{0} & \mathbf{e}_{1} & \mathbf{0} & \mathbf{e}_{2} & \mathbf{0} & \ldots & \mathbf{e}_{\frac{K_{p}}{2}}
\end{array}\right] .
\end{aligned}
$$

We have

$$
\mathbf{H}_{\text {aug }, n}^{\prime}=\left[\begin{array}{c}
\mathbf{F}_{o}^{\prime} \mathbf{h}_{n} \\
\mathbf{F}_{e}^{\prime} \mathbf{h}_{n}
\end{array}\right]=\left[\begin{array}{c}
\mathbf{F}_{o}^{\prime} \mathbf{h}_{n} \\
\Phi \mathbf{F}_{o}^{\prime} \mathbf{h}_{n}
\end{array}\right]
$$

where the two sub-matrices $\mathbf{F}_{o}^{\prime}, \mathbf{F}_{e}^{\prime}$ contain the odd and even rows of $\mathbf{F}^{\prime}$ respectively. Exploiting the tile structure (Fig. 2) the sub-matrices are connected by a diagonal matrix $\Phi=\operatorname{diag}\left(e^{\frac{-j 2 \pi t_{s} l_{0}}{K}}, \ldots, e^{\frac{-j 2 \pi t_{s} l_{L-1}}{K}}\right)$ as $\mathbf{F}_{e}^{\prime}=\Phi \mathbf{F}_{o}^{\prime}$ where $t_{s}=3$ is the tile separation.

The first stage of the algorithm is acquisition of the subspace spanned by the dominant eigenvectors of the auto-correlation matrix of channel vector $\hat{\mathbf{H}}_{\text {aug }}^{\prime}$. The auto-correlation matrix is not directly estimated. Rather, its decomposition via a delaysubspace tracker is estimated. The reason for using a tracking algorithm, instead of an averaging technique like the forwardbackward and spatial smoothing approach [9], is the irregular spacing of the pilot subcarriers over the frequency grid. With such as irregular pilot subcarrier spacing, techniques such as [9] cannot be easily used.

\section{A. Subspace learning}

The subspace tracking algorithm is as follows:

Initialize: $L_{m}$ - the upper limit on the number of paths,

$$
\mathbf{Q}_{0}=\left[\begin{array}{c}
\mathbf{I}_{L_{m}} \\
\mathbf{0}_{K_{p} \times L_{m}}
\end{array}\right], \mathbf{C}_{0}=\mathbf{I}_{L_{m}}, \mathbf{A}_{0}=\mathbf{0}_{K_{p} \times L_{m}} .
$$

For every OFDM symbol with pilot subcarriers update,

$$
\begin{aligned}
& \mathbf{Z}_{n}=\mathbf{Q}_{n-1}^{H} \hat{\mathbf{H}}_{\text {aug }, n}^{\prime} \\
& \mathbf{A}_{n}=\frac{1}{n}\left\{(n-1) \mathbf{A}_{n-1} \mathbf{C}_{n-1}+\hat{\mathbf{H}}_{\text {aug }, n}^{\prime} \mathbf{Z}_{n}^{H}\right\} \\
& \left.\mathbf{A}_{n}=\mathbf{Q}_{n} \mathbf{R}_{n} \quad \text { (QR decomposition }\right) \\
& \mathbf{C}_{n}=\mathbf{Q}_{n-1}^{H} \mathbf{Q}_{n} .
\end{aligned}
$$

Differing from [8] is the incorporation of the new information into the matrix. In (13), we have used linear averaging, in which the effect of a new symbol decreases with time, as opposed to applying a constant weight to the new value as in [8]. The reason for this, is that in a multi-user environment, the allocation scheme changes very rapidly, and hence the entire duration of the algorithm is several tens of OFDM symbols. We assume that within that period of time the delay-subspace remains constant ${ }^{2}$. In this scenario, it would be detrimental to use a constant weight for the new information, as it leads to gradual "memory loss" of information derived from past symbols which is still very relevant to the current estimation.

The matrix $\mathbf{Q}_{n}$ will eventually converge to the matrix of eigenvectors of the auto-correlation matrix $\mathbf{R}=$ $E\left\{\hat{\mathbf{H}}_{\text {aug }}^{\prime} \hat{\mathbf{H}}_{\text {aug }}^{\prime}\right\}$. The difference in Frobenius norm between consecutive eigenvector matrices is used as a metric to quantify the convergence. Once the metric goes below a predefined threshold, we declare convergence.

\section{B. ESPRIT-based multipath delay estimation}

Once the subspace tracking algorithm converges, the number of paths are estimated $(\hat{L})$ using the method given in [8] and delay subspace basis is derived as $\mathbf{Q}_{s}=\mathbf{Q}_{n}(:,[1: \hat{L}])$.

The multipath delays are estimated from the delay subspace basis using ESPRIT algorithm. The two shift invariant subspaces $\hat{\mathbf{U}}_{1}, \hat{\mathbf{U}}_{2}$ are derived from the estimated delay subspace basis as (pp. 1171-1175, [12]),

$$
\begin{aligned}
& \hat{\mathbf{U}}_{1}=\left[\begin{array}{lll}
\mathbf{I}_{\frac{K_{p}}{2}} & \mathbf{0}_{\frac{K_{p}}{2} \times \frac{K_{p}}{2}}
\end{array}\right] \mathbf{Q}_{s} \\
& \hat{\mathbf{U}}_{2}=\left[\begin{array}{lll}
\mathbf{0}_{\frac{K_{p}}{2} \times \frac{K_{p}}{2}} & \mathbf{I}_{\frac{K_{p}}{2}}
\end{array}\right] \mathbf{Q}_{s} .
\end{aligned}
$$

The subspaces $\mathbf{U}_{1}, \mathbf{U}_{2}$ are such that $\mathcal{S}\left\{\mathbf{U}_{1}\right\}=\mathcal{S}\left\{\mathbf{F}_{o}\right\}$ and $\mathcal{S}\left\{\mathbf{U}_{2}\right\}=\mathcal{S}\left\{\mathbf{F}_{e}\right\}=\mathcal{S}\left\{\Phi \mathbf{F}_{o}\right\}$.

The steps involved in multipath delays (tap locations) estimation are [12]

(a) Solve for the matrix $\mathbf{Y}$, such that $\hat{\mathbf{U}}_{1} \mathbf{Y}=\hat{\mathbf{U}}_{2}$

(b) The $\hat{L}$ delays are estimated as

$$
\hat{l}_{i}=\left\lceil\frac{\arg \left\{\lambda_{i}^{*}\right\} K}{2 \pi t_{s}}\right\rfloor \quad i=0,1, \ldots, \hat{L}-1
$$

where $\left\{\lambda_{i}\right\}_{i=1}^{\hat{L}}$ are the eigenvalues of $\mathbf{Y}$ and $\arg \left\{\lambda_{i}^{*}\right\}$ are in the range $[0,2 \pi)$. The delays are uniquely identified if $l_{L-1}<\frac{K}{t_{s}}$. We assume perfect synchronization and hence $\hat{l}_{0}=0$.

It is important to note that since we cannot assume the pilots are evenly spaced, we cannot produce two translational invariance matrices from $\mathbf{Q}_{s}$ as suggested in [6] by chopping off the first row to produce $\hat{\mathbf{U}}_{1}$ and chopping off the last row to produce $\hat{\mathbf{U}}_{2}$. The translational invariance can be achieved only by separating the even and odd locations representing the measurements of the first pilot in each tile, and the last pilot in each tile respectively separated by $t_{s}$ subcarriers.

\section{Error handling in ESPRIT-based delay estimation}

In order to increase the accuracy in the multipath delay estimation, we set the error margin around each estimated

\footnotetext{
${ }^{2}$ If the subchannel allocation for a user is not changed across multiple frames, then the path delay estimation can take advantage of this fact and average across frames
} 
delay and construct a vector of delays $\mathbf{d}_{e m}=\bigcup_{i=0}^{\hat{L}-1} \mathbf{d}_{i}$, where $\mathbf{d}_{0}=0, \mathbf{d}_{i}=\left[\hat{l}_{l}-\delta, . ., \hat{l}_{i}, . ., \hat{l}_{i}+\delta\right]$ and the error margin

$$
\delta=\left\lfloor\gamma\left(\frac{K_{p}}{\hat{L}-1}-1\right)\right\rfloor, \quad 0 \leq \gamma \leq 1 .
$$

The $\delta$ determines the number of paths that are estimated given the set of pilot subcarriers. $\gamma$ is a user-adoptable parameter.

In $q^{t h}$ slot, the channel gains $\hat{\mathbf{h}}_{q_{1}}$ at the delays $\mathbf{d}_{e m}$ on the first OFDM symbol are estimated by solving

$$
\hat{\mathbf{H}}_{l s, q_{1}}^{\prime}=\mathbf{F}_{1}^{\prime} \hat{\mathbf{h}}_{q_{1}}
$$

where $\mathbf{F}_{1}^{\prime}$ is the modified matrix obtained from the $K$-point DFT matrix by selecting rows corresponding to pilot indices $\mathcal{I}_{p}$ and columns corresponding to delays $\mathbf{d}_{e m}$. Similarly, the channel estimates $\hat{\mathbf{h}}_{q_{3}}$ are estimated. The metric $\mathbf{m}$ is then calculated as

$$
\mathbf{m}=\left|\frac{1}{2}\left(\hat{\mathbf{h}}_{q_{1}}+\hat{\mathbf{h}}_{q_{3}}\right)\right|^{2} .
$$

In the extended region around each estimated delay $\hat{l}_{i}$, we take the tap location with largest value of the metric as the improved estimate $\tilde{l}_{i}$ given by

$\tilde{l}_{i}=\arg \max _{-\delta \leq k \leq \delta}\left\{m\left(\hat{l}_{i}+k\right)\right\}$ for $1 \leq i \leq \hat{L}-1$ and $\tilde{l}_{0}=0$.

The metric used here is specifically designed for a fast fading channel in which the coherence time is an order of a symbol length. In that case, the correlation between the channel response of two symbols falls rapidly after a timeslot length. Therefore, averaging across symbols, further than a slot apart, would reduce the metric value at tap locations to a value that is indistinguishable from noise level. On the other hand, simply averaging the tap power across time would make low-power taps undistinguishable from the noise level, and would not exploit the small-range time correlation between symbols.

Setting the constant $\gamma=1$, pushes the error margin to the largest value, that still produces a single solution to the equation. However, our experiments show that a much lower constant, $\gamma=0.5$ is preferable. The reason being, the probability that a non-tap delay metric will surpass the tap-delay metric increases significantly as the error margin increases. The trade-off is hence between making the error margin large enough to include the location of the original channel tap, but not too large to make the tap-delay metric nearly undistinguishable from noise taps.

\section{Channel Interpolation}

Once the tap locations are estimated, the improved time domain channel estimates $\mathbf{h}_{l s, q_{i}}$ are obtained by solving

$$
\hat{\mathbf{H}}_{l s, q_{i}}^{\prime}=\mathbf{F}_{2}^{\prime} \hat{\mathbf{h}}_{l s, q_{i}} \quad i \in(1,3)
$$

where $\mathbf{F}_{2}^{\prime}$ is the modified matrix obtained from the $K$-point DFT matrix by selecting rows corresponding to pilot indices
$\mathcal{I}_{p}$ and columns corresponding to tap locations $\left\{\tilde{l}_{i}\right\}_{i=0}^{\hat{L}-1}$. The channel response on all the subcarriers of desired user is estimated as

$$
\hat{\mathbf{H}}_{q_{i}}=\mathbf{F}_{3} \hat{\mathbf{h}}_{l s, q_{i}} \quad i \in(1,3)
$$

where $\mathbf{F}_{3}$ is the modified matrix obtained from the $K$-point DFT matrix by selecting rows corresponding to subcarrier indices $\mathcal{I}$ and columns corresponding to tap locations $\left\{\tilde{l}_{i}\right\}_{i=0}^{\hat{L}-1}$.

The channel estimates for symbol $q_{2}$ is the simple average of the channel estimates of symbols $q_{1}$ and $q_{3}$

$$
\hat{\mathbf{H}}_{q_{2}}=\frac{1}{2}\left\{\hat{\mathbf{H}}_{q_{1}}+\hat{\mathbf{H}}_{q_{3}}\right\} \text {. }
$$

\section{Simulation And Results}

An OFDMA system is simulated with following parameters, center frequency $f_{c}=2.2 \mathrm{GHz}$, bandwidth $B=20 \mathrm{MHz}$, total number of subcarriers used $K=2048$, number of useful subcarriers $K_{u}=1680$, number of subchannels allocated to the desired user per slot $N_{s}=3$, length of cyclic prefix $L_{c p}=512$, upper limit on the number of paths $L_{m}=15$, $\gamma=0.5$, fade rate $f_{d}=240 \mathrm{~Hz}$.

The performance of the algorithm is evaluated by averaging over different channel realizations by selecting the multipath delays independently for the desired user, using normalized mean square error (NMSE) defined as

$$
\mathrm{NMSE}=\frac{E\left\{\sum_{i \in \mathcal{I}}\left|H_{i}-\hat{H}_{i}\right|^{2}\right\}}{E\left\{\sum_{i \in \mathcal{I}}\left|H_{i}\right|^{2}\right\}} .
$$

The performance of the proposed inter-tile based channel

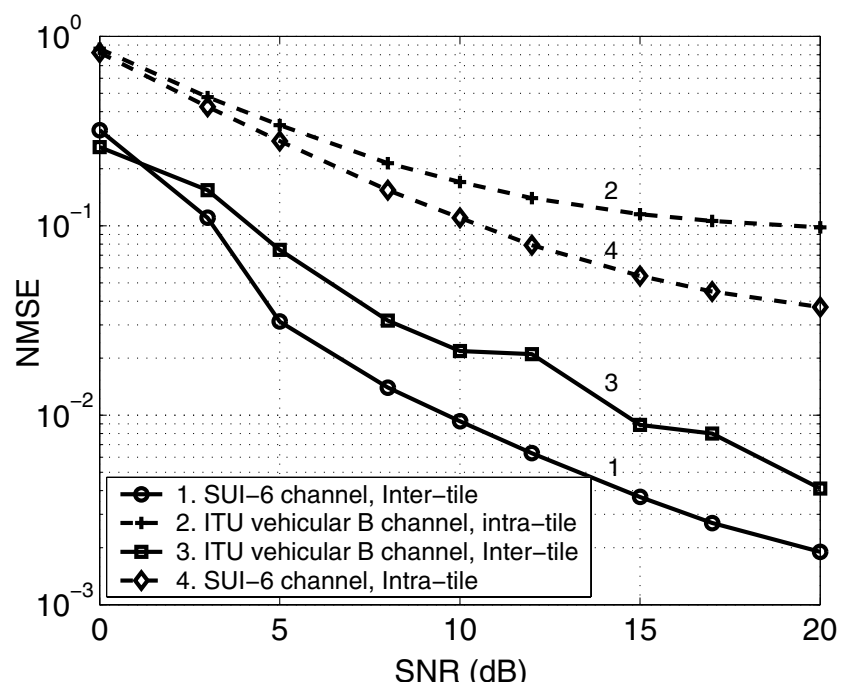

Fig. 3. NMSE comparison of intra-tile and inter-tile based estimators

estimation method is compared with existing intra-tile based method. The intra-tile based method estimates the channel at 
data subcarriers using the linear interpolation method given in [4]. The fig. 3 shows the NMSE plot comparing the intertile based method with the intra-tile based method for SUI-6 (3 paths) and ITU vehicular-B (6 paths) channel models. The intra-tile based method treats the channel estimates on pilots of each tile independently where as the inter-tile based method accounts for the frequency domain correlation between the channel estimates on pilots of different tiles in an OFDM symbol. The error floor in intra-tile based method (shown in dotted lines) is due to its inability to exploit frequency correlation on the pilot channel estimates of different tiles. The performance improvement of the inter-tile based method is $\sim 8 \mathrm{~dB}$ in NMSE compared to the intra-tile based method. The performance of the proposed estimator improves as the number of multipath components reduces.

\section{CONCLUSIONS}

In this paper, we have discussed a channel estimation algorithm for the uplink receiver of an OFDMA system. The proposed method exploits global channel information compared to existing algorithms which treat the channel information on a tile-by-tile basis. This is achieved by relying on the uplink tile structure which generates the shift invariance property in the signal space, enabling the usage of the ESPRIT algorithm to estimate the channel tap locations. Although requiring more intensive computations at the base station than straightforward intra-tile interpolation, the algorithm significantly reduces the estimation NMSE which leads to a reduced BER, and as a result decreases the need for numerous packet re-transmissions.

\section{REFERENCES}

[1] IEEE P802.16-2004, Standard for local and metropolitan area networks Part 16: Air Interface for Fixed Broadband Wireless Access Systems, 2004.

[2] I. Koffman and V. Roman, "Broadband wireless access solutions based on OFDM access in IEEE 802.16," in IEEE Communications Magazine, pp. 96-103, April 2002.

[3] J. J. van de Beek, O. Edfors, M. Sandell, S. K. Wilson, and P. O. Borjesson, "On channel estimation in OFDM systems," in Proceedings of IEEE VTC'95, vol.2, pp. 815-819, July 1995.

[4] S. Coleri, M. Ergen, A. Puri, and A. Bahai, "Channel estimation techniques based on pilot arrangement in OFDM systems," IEEE Trans. on Broadcasting, vol. 48, no. 3, pp. 223-229, September 2002.

[5] S. M. Kay, Fundamentals of Statistical Signal Processing Vol. I Estimation Theory, Prentice Hall, 1993.

[6] B. Yang, K. B. Letaief, R. S. Cheng, and Z. Cao, "Channel estimation for OFDM transmission in multipath fading channels based on parametric channel modeling," IEEE Trans. on Communications, vol. 49, no. 3, pp. 467-479, March 2001.

[7] M. R. Raghavendra and K. Giridhar, "Improving channel estimation in OFDM systems for sparse multipath channels", in IEEE Signal Processing Letters, vol. 12, no. 1, pp. 52-55, January 2005.

[8] O. Simeone, Y. Bar-Ness, and U. Spagnolini, "Pilot-based channel estimation for OFDM systems by tracking the delay-subspace," IEEE Trans. on Wireless Communications, vol. 3, no. 1, pp. 315-325, January 2004.

[9] S. U. Pillai and B. H. Kwon, "Forward/backward spatial smoothing techniques for coherent signal identification," IEEE Trans. on Acoustics, Speech, and Signal Processing, vol. 37, no. 1, pp. 8-15, January 1989.

[10] R. Roy and T. Kailath, "ESPRIT - Estimation of signal parameters via rotational invariance techniques," IEEE Trans. on Acoustics, Speech, and Signal Processing, vol. 37, no. 7, pp. 984-995, July 1989.

[11] R. Steele, Mobile Radio Communications, IEEE Press, 1992.
[12] H. L. Van Trees, "Optimum Array Processing, part IV of Detection, Estimation, and Modulation Theory", John Wiley, 2002. 